\title{
Prognostic nomogram on clinicopathologic features and serum indicators for advanced non-small cell lung cancer patients treated with anti-PD-1 inhibitors
}

\author{
Rong Chai ${ }^{1, \wedge}$, Yinxing Fan ${ }^{1,2 \#}$, Jiayi Zhao ${ }^{3 \#}$, Fan $\mathrm{He}^{1 \#}$, Jianong $\mathrm{Li}^{1}$, Yiping Han ${ }^{1}$ \\ ${ }^{1}$ Department of Respiratory and Critical Care Medicine, Changhai Hospital, Navy Military Medical University, Shanghai, China; ${ }^{2}$ Zhenjiang \\ Medical District, General Hospital of Eastern Theater Command, Zhenjiang, China; ${ }^{3}$ Department of General Practice Teaching and Research \\ Office, Changhai Hospital, Navy Military Medical University, Shanghai, China \\ Contributions: (I) Conception and design: R Chai, Y Fan, J Zhao, Y Han; (II) Administrative support: None; (III) Provision of study materials or \\ patients: None; (IV) Collection and assembly of data: R Chai, F He, J Li; (V) Data analysis and interpretation: R Chai, Y Fan; (VI) Manuscript \\ writing: All authors; (VII) Final approval of manuscript: All authors. \\ \#These authors contributed equally to this work. \\ Correspondence to: Yiping Han. Department of Respiratory and Critical Care Medicine, Changhai Hospital, Navy Military Medical University, No. \\ 168 Changhai Rd, Yangpu District, Shanghai, China. Email: yphan2006@163.com.
}

Background: Immune checkpoint inhibitors (ICIs) have appeared as a promising therapy regimen for nonsmall cell lung cancer (NSCLC), but with an unsatisfying therapeutic response and inefficiency of a single predictive biomarker in patients' selection.

Methods: Central data of clinicopathologic features, peripheral blood indicators, and treatment records were collected in advanced NSCLC patients accepting PD-1 inhibitors in Changhai Hospital from July 2016 to September 2019. The OS probability nomogram was developed according to Akaike Information Criterion (stepAIC) selected factors. The predictive accuracy of the nomogram was assessed by discrimination and calibration. C-index and decision curve analysis were used to compare with the previously reported model (Botticelli Model). Computers resampling 500 times (Bootstrap 500 times) were performed to validate the model internally. According to the nomogram-based total point scores (TPS), we divided patients into different risk groups.

Results: A total of 110 patients were enrolled in this study. Six predictors, including liver metastasis, Eastern Cooperative Oncology Group Performance Status (ECOG PS), second- or third-line immunotherapy, baseline levels of CRP, cytokeratin 19 fragment (CYFRA21-1), were selected to set up the nomogram. The C-index of the current nomogram was 0.81 (95\% CI: 0.72-0.80), keeping the same accuracy as the earlier one. Calibration plots showed slight underestimation in patients with predictive mortality $<44 \%$ at 12 months and overestimation in patients with predictive mortality $>44 \%$. Decision curve analysis showed that the current nomogram was with a higher net benefit rate than the earlier model. According to the cut-off points of TPS, patients were divided into three subgroups: low risk (TPS $\leq 118$ ), intermediaterisk $(118<$ TPS $\leq 189)$, and high risk (TPS >189). A significant OS difference was observed among subgroups. Median OS was 6.6, 4.5, 1.3 months, respectively.

Conclusions: We proposed a novel nomogram model on easily available and inexpensive clinicopathologic features, peripheral blood indicators which is beneficial in individual risk assessment for advanced NSCLC patients before receiving PD-1 inhibitors, and assisting clinicians in accurately determining therapeutic decisions.

Keywords: Nomogram; immunotherapy; clinicopathologic characteristics; peripheral blood biomarkers; advanced non-small cell lung cancer (advanced NSCLC)

Submitted May 15, 2020. Accepted for publication Jul 22, 2020.

doi: 10.21037/atm-20-4297

View this article at: http://dx.doi.org/10.21037/atm-20-4297

\footnotetext{
^ ORCID: 0000-0002-6816-6416.
} 


\section{Introduction}

The latest epidemiological data indicate that lung cancer still links to the second most common cancer with the highest mortality rate on a global scale (1). As a malignant tumor with complex histological types, about $85 \%$ of lung cancer cases belong to non-small cell lung cancer cells (NSCLC) with a five-year survival rate of $19 \%(2,3)$.

In the past two decades, significant evolvement of treatment in NSCLC has been made with the introduction of several lines of small molecule tyrosine inhibitors in patients with EGFR, ALK, ROS1 mutations and the discovery of potent KRAS locking inhibitors. Similarly, with an impressive clinical benefit and tolerable adverse effect, immune checkpoint inhibitors (ICIs) have revolutionized the management of advanced NSCLC patients without actionable oncogenic drivers, improving the five-year survival rate to $16 \%$ (4). The fast-growing number of immunotherapeutic patients and undesirable response rates underline the importance of finding predictive biomarkers to aid clinical decision making. Widely tested as the expression of the PD-L1 on tumor cells or immune cells determined by immunohistochemistry has, PD-L1 is a suboptimal predictive biomarker due to the dynamic changes of PD-L1 expression over time, intra-tumoral heterogeneity, absence of a uniform guideline for test method and positive clinical threshold (5-7). Another extensively studied predictive biomarker tumor mutation burden (TMB) reflects the expression level of unstable genome induced neoantigens (8), which is promising in clinical application but with the limitation of high cost and shortage of consensus on detection methods $(9,10)$. Other potential predictors such as tumor-infiltrating lymphocytes (11), tumor-specific genomics (12), were explored but have not yet been proven to be feasible in clinical practice.

Based on the multifactorial nature of cancerimmune interactions and ever-changing tumor immune microenvironment (TME), combining parameters are supposed to be the future of response prediction to immunotherapies. Blank (13) proposed a conception of "cancer immunogram" that enhanced T cell activity was the mechanism of the ultimate effect of immunotherapies. Seven dimensions may be the first framework of the "cancer immunogram." Using relevant parameters to set up a prognostic model may be an essential direction for the supervision of immunotherapeutic effects in patients with advanced NSCLC. Botticelli (14) developed a nomogram based on three clinicopathological factors, including liver and lung metastases and Eastern Cooperative Oncology
Group Performance Status (ECOG PS), to predict overall survival (OS) probability in NSCLC patients accepting nivolumab.

Before, it was easily available, inexpensive, and allowing longitudinal observation that promoted to the increasing research of peripheral biomarkers such as neutrophil-toLymphocyte ratio (NLR), platelet-to-lymphocyte ratio (PLR) (15), serum tumor markers (16) and inflammatory parameters (17). The study aimed to develop an OS probability predictive model to evaluate individual risk in advanced NSCLC patients before receiving PD-1 inhibitors by using easily accessible clinicopathological parameters and serum biomarkers. We present the following article in accordance with the TRIPOD reporting checklist (available at http://dx.doi.org/10.21037/atm-20-4297).

\section{Methods}

\section{Patients}

We reviewed the electronic medical records of all patients with recurrent or advanced (stage IIIB to IV) NSCLC who had accepted PD-1 inhibitors (nivolumab or pembrolizumab) monotherapy or combined-therapy as firstline, maintenance, second-line, or further line regimen at Changhai Hospital between July 2016 and September 2019. From this review, we found a total of 126 patients receiving the injection of PD-1 inhibitors. Patients who received injection of PD-1 inhibitor with complete clinical data, therapeutic and following-up information were enrolled into data analysis. Patients with autoimmune diseases, other malignancies, and symptomatic interstitial lung diseases were excluded. According to the criteria, 110 individuals were included in data analysis. The study was conducted in accordance with the Declaration of Helsinki (as revised in 2013), and was reviewed and approved by the ethics committee of Changhai Hospital. All patients had signed informed consent.

\section{Data acquisition}

Data on clinicopathological features, peripheral blood indicators, and treatment records were extracted from the electronic inpatient record system or acquired by telephone. Follow-up, including gender, age, ECOG PS, smoking status, maximum lesion diameter, metastatic sites, EGFR/ALK/ROS1 mutation status, tumor staging, PDL1 expression level, and other clinical pathological features were gathered. The baseline complete blood cell count 
and its ratio, lactate dehydrogenase (LDH), C-reactive protein (CRP), albumin, carcinoembryonic antigen (CEA), cytokeratin 19 fragment (CYFRA21-1) and other serum indicators; immunotherapeutic regimens, commencement time of PD-1 inhibitors.

All data were last updated in December 2019. Complete blood count (CBC) was performed before the first injection of PD-1 inhibitor (baseline) and at the followup time point. Serum CRP concentration was determined with immunoturbidimetry. By immunoradiometric assay, CYFRA21-1 was measured. All operations and tests are carried out in accordance with the kit guidelines. Calculate formula: nutritional prognosis index $(\mathrm{PNI})=$ albumin $(\mathrm{g} / \mathrm{L})+5 \times$ lymphocyte counts $\times 10^{9} / \mathrm{L} ; \mathrm{NLR}=\mathrm{ANC} / \mathrm{ALC}$; PLR = PLT/ALC; monocytes-to-lymphocyte ratio (MLR) $=$ AMC/ALC. ANC, ALC, PLT and AMC were the counts of absolute neutrophils, absolute lymphocytes, platelet and monocytes, respectively.

\section{Treatment and efficiency assessment}

For the reason of the respective study, not all patients received standard injection does of nivolumab $(3 \mathrm{mg} / \mathrm{kg}$ every 2 weeks) or pembrolizumab (200 mg every 3 weeks). Part of patients adopted combined therapy with antiangiogenic medicines, radiotherapy, or chemotherapies. Chest CT was undergone at every $8-9$ weeks to evaluate the radiological response of tumors. The best response pattern and disease progression were evaluated according to the RESIST1.1. The definition of progression-free survival (PFS) and OS were following earlier reports. Patients without observed clinical or radiographic disease progression or who were still alive were censored on the date of the last follow-up.

\section{Statistical analysis}

Mean standard deviation or median (min-max) was used to describe continual variables. Categorical variables were presented as percentages. COX univariate and multivariate survival analysis were applied to evaluate the impact of peripheral blood parameters and clinicopathological factors on PFS and OS. Then, in univariate analysis, OSrelated variables $(\mathrm{P}<0.05)$ were included in Stepwise Akaike Information Criterion (stepAIC) analysis to select out factors for the establishment of an OS probability predictive nomogram. C-index of discrimination and calibration curves were presented to qualify the predictive accuracy of the nomogram (18). And 500 bootstrap re-samplings were performed to validate this model (19). C-index and decision curve analysis (DCA) was executed to compare the predictive accuracy and net benefit rates between the current model and the Botticelli model (14). At last, X-tile software was applied to determine the cut-off points of the nomogram-based total point scores (TPS). Kaplan-Meier curves and Log-rank tests were used to qualify the performance of current model in stratifying the risk of patients.

All statistical analyses were performed through the Empower Stats (http://www.empowerstats.com, X\&Y Solutions, Inc., Boston, MA, USA) and R software 3.6.2 (http://www.r-project.org). A two-tailed P value of $<0.05$ was considered statistically significant.

\section{Results}

\section{Patient characteristics}

The average age of patients was $63.8 \pm 9.7$ years, and $80.0 \%$ were males. Former or current smokers make up $63.6 \%$ of the subjects. Half of the patients were lung squamous carcinoma, forty-nine cases with adenocarcinoma, six cases with large cell carcinoma, or mixed adenosquamous cancer. Eighty-nine $(80.9 \%)$ patients had ECOG PS 0 or 1 , and $11.8 \%$ involved liver metastasis. There were 39 (35.5\%), $24(21.8 \%)$, and $47(42.7 \%)$ patients received immunotherapy as first-line, second-line, or $\geq$ three-line treatment, respectively. Sixty-nine patients accepted PD-1 inhibitor combining with radiotherapy, antiangiogenic drugs or chemotherapy, and mono-immunotherapy was applied in 41 patients. Other baseline clinicopathological factors were shown in Table 1.

Patients had an average baseline level of CRP $19.1 \mathrm{mg} / \mathrm{L}$ and CYFRA21-1 $6.5 \mathrm{ng} / \mathrm{mL}$. Other baseline levels of serum parameters were presented in Table 2. At the last date of follow-up, the median OS was 5.5 months, thirty-eight patients died.

\section{Univariate and multivariate COX survival analysis of the OS}

COX univariate analysis showed that baseline level of ANC, NLR, PLR, CRP, CEA, CYFRA21-1 and treatment line, ECOG PS and smoking were related to $\mathrm{OS}(\mathrm{P}<0.05$, Table 3). Then, multivariate analysis through stepwise univariate analysis indicated that smoking was an independent protective factor for OS (HR $=0.11,95 \%$ CI: 
Table 1 Patients clinicopathological characteristics $(\mathrm{n}=110)$

\begin{tabular}{|c|c|}
\hline Variables & $\mathrm{N}(\%)$ \\
\hline Age (years) & $63.8 \pm 9.7$ \\
\hline \multicolumn{2}{|l|}{ Gender } \\
\hline Male & $88(80.0)$ \\
\hline Female & $22(20.0)$ \\
\hline \multicolumn{2}{|l|}{ Smoking } \\
\hline No & $40(36.4)$ \\
\hline Yes & $70(63.6)$ \\
\hline \multicolumn{2}{|l|}{ TNM stage } \\
\hline III & $25(22.7)$ \\
\hline IV & 85 (77.3) \\
\hline \multicolumn{2}{|l|}{ Pleural metastasis } \\
\hline No & $75(68.2)$ \\
\hline Yes & $35(31.8)$ \\
\hline \multicolumn{2}{|l|}{ Lung metastasis } \\
\hline No & $59(53.6)$ \\
\hline Yes & $51(46.4)$ \\
\hline \multicolumn{2}{|l|}{ Bone metastasis } \\
\hline No & $76(69.1)$ \\
\hline Yes & $34(30.9)$ \\
\hline \multicolumn{2}{|l|}{ Liver metastasis } \\
\hline No & 97 (88.2) \\
\hline Yes & $13(11.8)$ \\
\hline \multicolumn{2}{|l|}{ Braine metastasis } \\
\hline No & $96(87.3)$ \\
\hline Yes & $14(12.7)$ \\
\hline \multicolumn{2}{|l|}{ Histological types } \\
\hline Squamous cell carcinoma & $55(50.0)$ \\
\hline Adenocarcinoma & $49(44.5)$ \\
\hline Other & $6(5.5)$ \\
\hline \multicolumn{2}{|l|}{ EGFR mutation } \\
\hline No & $100(90.9)$ \\
\hline Yes & $10(9.1)$ \\
\hline \multicolumn{2}{|l|}{ ROS1 mutation } \\
\hline No & $108(98.2)$ \\
\hline Yes & $2(1.8)$ \\
\hline
\end{tabular}

Table 1 (continued)
Table 1 (continued)

\begin{tabular}{lc}
\hline Variables & $\mathrm{N}(\%)$ \\
\hline ECOG PS & $89(80.9)$ \\
0 or 1 & $21(19.1)$ \\
2 & \\
Treatment line & $39(35.5)$ \\
First-line & $24(21.8)$ \\
Second-line & $47(42.7)$ \\
$\geq$ Three-line & \\
Mono-immunotherapy & $69(62.7)$ \\
No & $41(37.3)$ \\
Yes
\end{tabular}

Table 2 Baseline level of peripheral parameters

\begin{tabular}{|c|c|}
\hline Parameters & Mean $\pm \mathrm{SD} /$ median $(\mathrm{Q} 1-\mathrm{Q} 3)$ \\
\hline ANC $\left(\times 10^{9} / L\right)$ & $5.1 \pm 2.7$ \\
\hline AMC $\left(\times 10^{9} / L\right)$ & $0.7 \pm 0.5$ \\
\hline $\operatorname{ALC}\left(\times 10^{9} / \mathrm{L}\right)$ & $1.4 \pm 0.5$ \\
\hline NLR & $4.2 \pm 3.0$ \\
\hline MLR & $0.6 \pm 0.5$ \\
\hline $\operatorname{PLT}\left(\times 10^{12} / \mathrm{L}\right)$ & $269.1 \pm 131.4$ \\
\hline PLR & $217 \pm 138$ \\
\hline $\mathrm{Hb}(\mathrm{g} / \mathrm{dL})$ & $123 \pm 20$ \\
\hline $\mathrm{CRP}(\mathrm{mg} / \mathrm{L})$ & $19.1(1.2-250.0)$ \\
\hline CEA (ng/mL) & $4.7(1.1-1,500.0)$ \\
\hline CYFRA21-1 (ng/mL) & $6.5(0.6-100.0)$ \\
\hline LDH (IU/L) & $217.8 \pm 87.0$ \\
\hline PNI & $44.1 \pm 6.3$ \\
\hline Alb (g/L) & $37.6 \pm 4.3$ \\
\hline
\end{tabular}

NLR, neutrophil-to-lymphocyte ratio; MLR, monocytesto-Iymphocyte ratio; CRP, C-reactive protein; CEA, carcinoembryonic antigen; CYFRA21-1, cytokeratin 19 fragment; $\mathrm{LDH}$, lactate dehydrogenase; PNI, nutritional prognosis index. 
Table 3 Univariate and multivariate COX analysis for OS

\begin{tabular}{|c|c|c|c|c|c|}
\hline Variables & Subgroup & \multicolumn{2}{|c|}{ Univariate analysis } & \multicolumn{2}{|c|}{ Multivariate analysis } \\
\hline Gender & Female & $1.4(0.7-2.8)$ & 0.389 & - & - \\
\hline Smoking & Yes & $0.4(0.2-0.8)$ & 0.004 & $0.1(0.0-0.3)$ & 0.001 \\
\hline Pleural metastasis & Yes & $1.8(0.95-3.56)$ & 0.071 & - & - \\
\hline Bone metastasis & Yes & $1.72(0.88-3.37)$ & 0.111 & - & - \\
\hline Liver metastasis & Yes & $2.80(1.27-6.19)$ & 0.011 & - & - \\
\hline Brain metastasis & Yes & $0.95(0.33-2.70)$ & 0.924 & - & - \\
\hline Histological type & LUAD vs. LUSC & $1.42(0.72-2.80)$ & 0.311 & $10.10(2.17-47.01)$ & 0.003 \\
\hline Age & & $1.03(1.00-1.07)$ & 0.082 & - & - \\
\hline ANC & & $1.15(1.03-1.29)$ & 0.012 & - & - \\
\hline CEA & & $1.00(1.00-1.00)$ & 0.005 & - & - \\
\hline CYFRA21-1 & & 1.01 (1.00-1.02) & 0.021 & $1.04(1.01-1.06)$ & 0.002 \\
\hline ALC & & $0.67(0.33-1.34)$ & 0.377 & - & - \\
\hline NLR & & $1.13(1.05-1.21)$ & 0.001 & - & - \\
\hline MLR & & $1.49(1.02-2.18)$ & 0.038 & - & - \\
\hline PLT & & $1.00(1.00-1.00)$ & 0.311 & $0.98(0.97-1.00)$ & 0.049 \\
\hline PNI & & $0.97(0.94-1.01)$ & 0.207 & - & - \\
\hline \multirow[t]{2}{*}{ Treatment line } & 2nd vs. 1st-line & $2.64(0.94-1.01)$ & 0.078 & $19.75(2.56-152.2)$ & 0.004 \\
\hline & 3rd vs. 1st-line & $3.62(1.37-9.60)$ & 0.010 & 36.9 (5.11-266.56) & $<0.001$ \\
\hline ECOG & 2 vs. $\leq 1$ & $6.16(3.12-12.16)$ & $<0.001$ & - & - \\
\hline
\end{tabular}

OS, overall survival; NLR, neutrophil-to-lymphocyte ratio; MLR, monocytes-to-lymphocyte ratio; CRP, C-reactive protein; CEA, carcinoembryonic antigen; CYFRA21-1, cytokeratin 19 fragment; LDH, lactate dehydrogenase; PNI, nutritional prognosis index.

0.02-0.51, $\mathrm{P}=0.005)$, but baseline level of CRP $(\mathrm{HR}=1.02$, 95\% CI: 1.01-1.04, $\mathrm{P}=0.007)$, CYFRA21-1 (HR $=1.04,95 \%$ CI: $1.00-1.06, \mathrm{P}=0.002)$, PLT (HR=0.98, 95\% CI: $0.97-$ $1.00, \mathrm{P}=0.0493)$ and second-line treatment $(\mathrm{HR}=19.75$, 95\% CI: 2.56-152.22, $\mathrm{P}=0.004)$ or third-line treatment (HR $=36.9,95 \%$ CI: $5.11-266.56, \mathrm{P}<0.001)$ were significantly associated with shortened OS.

Establishment and validation of OS probability prediction nomogram

In the COX univariate analysis, variables related to OS 


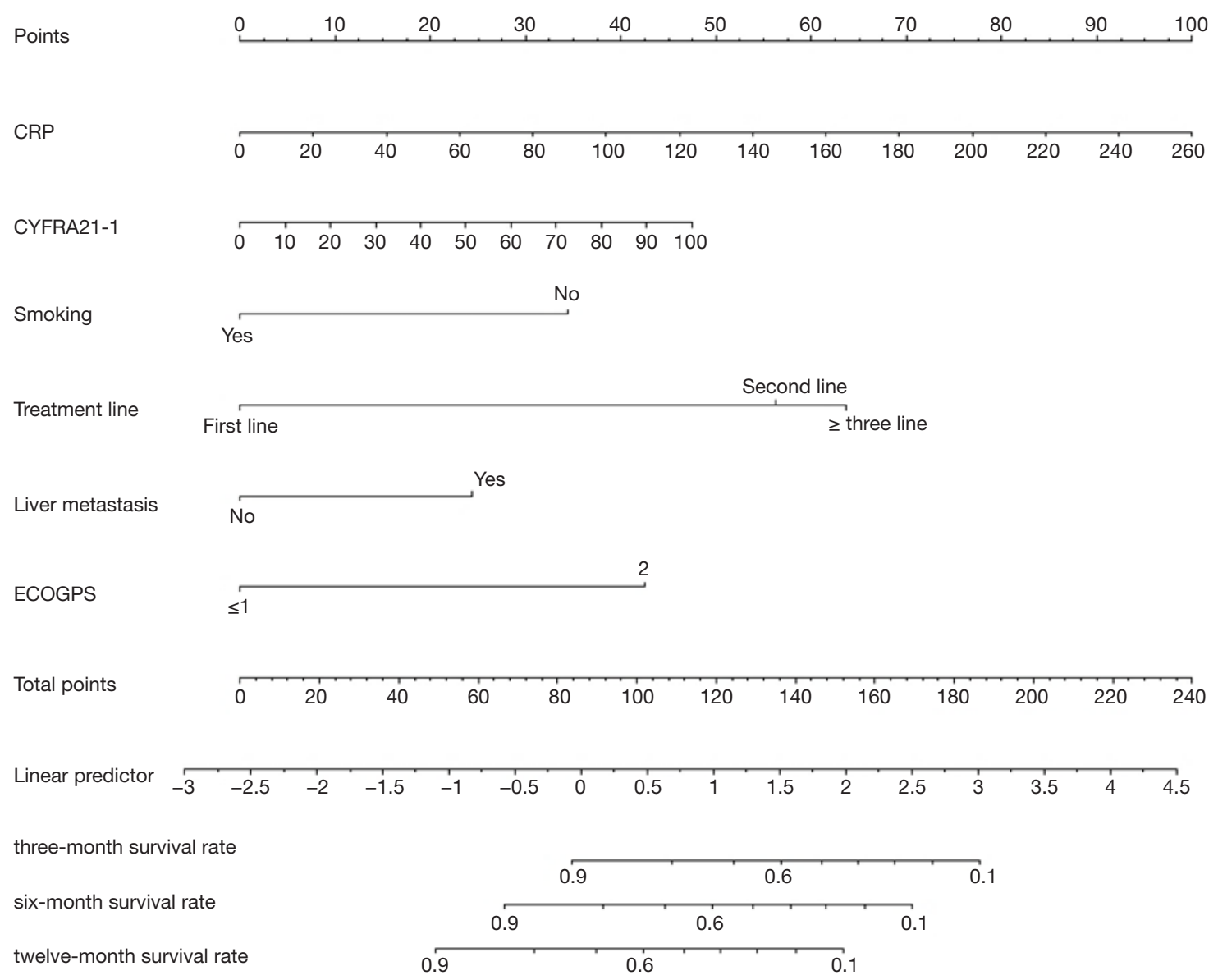

Figure 1 Prognostic nomogram of OS probability at 3-, 6- and 12-month in advanced NSCLC patients treated with PD-1 inhibitors For each factor, the number of corresponding risk points can be determined by delineating a vertical line from the prognostic factor to the points raw (0 to 100), and sum the corresponding risk points to determine the total points for each patient. The corresponding probability of survival at 3,6, and 12 months can be obtained by drawing a straight line from the total points axis to the OS probability axis. OS, overall survival; NSCLC, non-small cell lung cancer.

$(\mathrm{P}<0.05)$ were screened through stepwise AIC regression. Smoking, liver metastasis, treatment lines, ECOG PS, the baseline level of CYFRA21-1 and CRP were integrated into the dynamic prediction nomogram model to calculate survival probability at 3,6 , and 12 months in advanced NSCLC patients treated with PD-1 inhibitors (Figure 1).

Each prognostic parameter has a corresponding number of risk points, which can be obtained by delineating a vertical line from the prognostic factor to the points raw. Then, the corresponding risk points of each parameter were summed to determine the total points scored. Finally, from the total points axis, a vertical line can be drawn towards the OS probability axis to obtain the 3-, 6- and 12-month OS probability for a specific patient. For example, a smoking $(0$ point) patient with ECOG PS 1 (0 points), liver metastasis ( 25 points), a baseline level of CRP $100 \mathrm{mg} / \mathrm{L}$ (39 points), CYFRA21-1 $40 \mathrm{ng} / \mathrm{L}$ (15.5 points) and accepting PD-1 inhibitors as second-line therapy (58 points), the sum of the total risk points was 137.5 points. By drawing a vertical line down the "3-, 6- and 12-month survival probability" axis, the survival probability of 3-, 6and 12 -month was $58 \%, 40 \%$, and $12 \%$, respectively.

C-index for the current OS model was 0.81 (95\% CI: 0.72-0.90), showed it had distinguished discrimination. According to the calibration curve (Figure 2), among patients with actual mortality of greater than $44 \%$, the model would overestimate mortality risk. For example, some individuals in whom the model predicted mortality risk of $60 \%$ (actual mortality of approximately 50\%) might improperly refuse immunotherapy due to the high estimated 


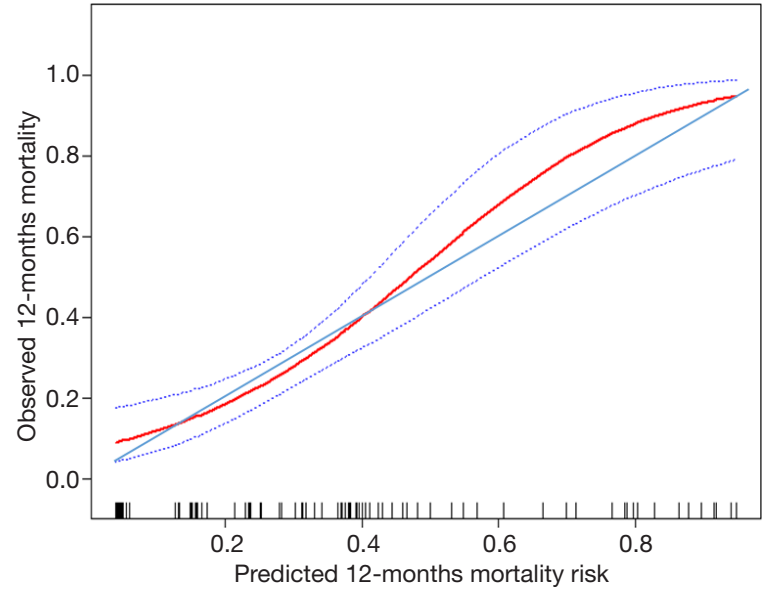

Figure 2 Calibration plot of predicted mortality-probability against the observed mortality-probability at 12 months. The red regression curve is plotted to demonstrate the general trend; the dashed curve shows the $95 \%$ CI of the calibration curve; the blue line shows the ideal calibration line.

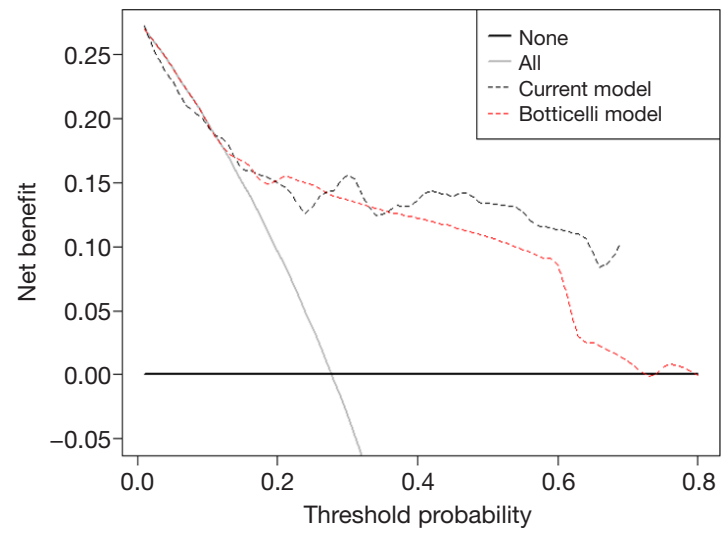

Figure 3 Decision curve analysis for 6-month survival black dotted line: current nomogram; red dotted line: Botticelli nomogram. Y-axis: net benefit $=$ total benefits (true positives) - harms (false positives). The straight grey line represents the assumption that all patients will die at six months, and the black horizontal line represents the assumption that no patients will die at six months.

risk of death and low expectation of therapeutic benefit. However, among patients with actual mortality of lower than $44 \%$, the model would underestimate mortality risk. For example, some individuals in whom the model predicted mortality risk of $30 \%$ (actual mortality of approximately $35 \%)$ might improperly accept immunotherapy due to the low estimated risk of death and high expectation of therapeutic benefit. Therefore, the current model had an

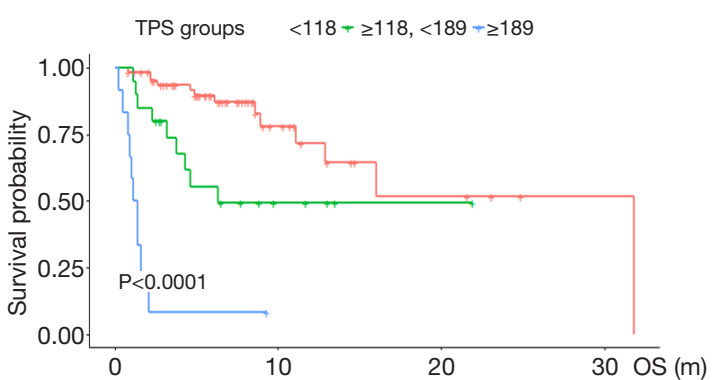

Figure 4 Kaplan-Meier curves for three subgroups based on the predictors from the nomogram. The red curve, green curve, and blue curve represent the group of TPS $\leq 118,118<$ TPS $\leq 189$, and TPS $>189$, respectively. The vertical axis is the survival rate, and the horizontal axis is over survival time (month). TPS, total point score.

adequate calibration ability, but using the model would lead some patients to refuse or accept immunotherapy, impairing benefits from that treatment inappropriately.

\section{Comparison of current nomogram and reported nomogram}

Comparison of the C-index of current nomogram model 0.81 (95\% CI: 0.72-0.90) with the reported model (Botticelli model) 0.76 (95\% CI: 0.68-0.81) presented no statistical difference indicating that the current model kept the same predictive accuracy as the Botticelli model. Decision curve analysis for 6-months survival (Figure 3) revealed that the current nomogram had a higher net benefit rate than the Botticelli nomogram, implying that patients could benefit from using the current model to guide clinical treatment decisions.

\section{Performance of the nomogram in stratifying patient risk}

Patients were divided into three subgroups according to the cut-off value of nomogram-based total score (TPS): low-risk (TPS $\leq 118,65$ cases), intermediate-risk (118< TPS $\leq 189,20$ cases), and high-risk (TPS $>189,12$ cases). A significant OS difference was observed among subgroups. Median OS was 6.6, 4.5, 1.3 months, respectively. KaplanMeier survival curve analysis manifested that patients in the high-risk group were linked to a shortened OS (Figure 4).

\section{Discussion}

ICIs with significant treatment effects and tolerable adverse events had developed a new standard management pattern for NSCLC patients. And there is an urgent need 
for predictive markers to precisely select immunotherapy optimal individuals. In the study, we used the easily accessible clinicopathological characteristics and serum parameters to establish a survival prognostic nomogram model for advanced NSCLC patients treated with PD-1 inhibitors. The nomogram model with superb predictive accuracy and discriminative ability could be applied to estimate individual risk for advanced NSCLC patients before the commencement of immunotherapy and assist in the decision-making process in clinical practice.

The establishing indicators between the current and reported models (14) were diverse for the different factors screening methods. Another reason was that our study included peripheral biomarkers. Comparison of the C-index between current nomogram (C-index 0.81, 95\% CI: $0.72-0.90)$ and reported nomogram (C-index 0.76 , 95\% CI: 0.65-0.85) implied that the predictive accuracy of current model kept the similar accuracy to reported model. Nevertheless, the current model was based on the real-world data of advanced NSCLC patients who received diversely immunotherapeutic strategies. The reported model was based on data of advanced NSCLC patients who received nivolumab as second-line therapy. The current nomogram might have a larger applicable population. Additionally, decision curve analysis for 6-month survival revealed a higher net benefit rate of the current model than reported one.

As a classic indicator for patient behavioral status, clinical trials and real-world studies all had demonstrated that ECOG PS $\geq 2$ was an independent predictor for dismal prognosis in NSCLC patients accepting systemic therapies $(20,21)$. Hence, the ECOG PS score needs to be routinely assessed in the clinical decision-making process. Studies reported that receiving Pembrolizumab combined with platinum-based chemotherapy as a first-line regimen was the optimal management for advanced NSCLC patients, and with the backward-shift of treatment lines benefit from immunotherapy would be impaired $(22,23)$. Our study also showed that the backward shift of treatment lines was paralleled to an increased prognostic risk score indicating the moment of adopting ICIs might impact clinical outcomes.

COX univariate and multivariate survival analysis manifested that tobacco exposure history was an independent protective factor for NSCLC patients adopting ICIs. The potential mechanism was that tobacco carcinogen-related mutagenesis was linked to elevated nonsynonymous mutation burden promoting the expression of neoantigens, which makes tumor cells more immunogenic, improving the recognition ability and sensitivity of $\mathrm{T}$ cells (24). Another possible mechanism was that tobacco exposure could shape chronic inflammation microenvironment to recruit tumorinfiltrating lymphocytes (TILs) and release interferon- $\gamma$ (IFN- $\gamma$ ) inducing PD-L1 expression and enhancing the stability of the PD-L1. As a routinely examined clinical marker, elevated serum level of CRP could reflect the host's chronic inflammatory status, and immune response to the tumor. In the study, baseline CRP level was positively correlated with patients' risk scores. CRP could be through IL-6/JAK/STAT3 signal pathway to promote cancer immune evasion (25).

Previous data demonstrated that NSCLC patients with liver metastasis had a reduced 3-year survival rate, inadequate treatment response, and shortened PFS in immunotherapy (26), and our study had observed the same association. Incomplete activation of CD8+T cells, capturing and clear of activated CD8+T cells could induce liver-related peripheral immune tolerance (27). Also, Bamboat (28) observed that difference in dendritic cell secretions in peripheral blood and liver was a potential interpretation of the diverse quantity and activity of cytotoxic $\mathrm{T}$ cells in the liver and circulation.

Serum tumor markers such as CEA and CYFRA 21-1 had been identified as useful prognostic and longitudinal monitoring biomarkers in NSCLC patients receiving chemotherapy (29) and targeted therapy (30). Recently, studies also found that serum level of CYFRA21-1 or CEA could assist in predicting immunotherapy efficacy (16). Our data showed that baseline levels of CEA and CYFRA21-1 were positively correlated with the patient's risk score before immunotherapy. CEA and CYFRA21-1 both are commonly used clinical indicators. The serum concentration of CYFRA21-1 is particularly elevated in the carcinoid tumors and LUSC (31). In this study, only CYFRA21-1 was selected as a predictive model construction factor, which may be related to the larger proportion of males and LUSC patients in this study.

Given the retrospective nature of the study, there were certain limitations. Firstly, as a retrospective study cannot exclude all potential biases. Secondly, many studies were inclined to transfer continuous parameters to binary variables, but we did not adopt the usual conversion. Moons (19) deemed that converting continuous variables into binary variables might lead to loss of major information and reduce the test power. Thirdly, only monocentric data of advanced NSCLC patients receiving PD-1 inhibitors were used to develop the nomogram with small sample sizes and imbalanced male-to-female ratio. Further larger scale, 
multi-center prospective studies, and external validation should be performed to check whether these results were suitable for the general population. At last, this study only focused on the prognostic function of clinicopathological characteristics and routinely used serum parameters in NSCLC patients treated with PD-1 inhibitors. Other broadly explored predictors such as PD-L1 and TMB were not included in the analysis.

In conclusion, the nomogram based on easily accessible clinicopathological characteristics and serum parameters was a simple and inexpensive prognostic tool for advanced NSCLC patients treated with PD-1 inhibitors, which could be adopted in individual risk assessment before patients receiving PD-1 inhibitors as well as assisting clinicians in making optimal therapeutic decisions.

\section{Acknowledgments}

We thank the staff of the Department of Respiratory and Critical Care Medicine, Shanghai Changhai Hospital (Shanghai, China), for assisting in data management and research conditions.

Funding: The present study was supported by the Shanghai Scientific Research Projects (grant No. 19411970600).

\section{Footnote}

Reporting Checklist: The authors have completed the TRIPOD reporting checklist. Available at http://dx.doi. org/10.21037/atm-20-4297

Data Sharing Statement: Available at http://dx.doi. org/10.21037/atm-20-4297

Conflicts of Interest: All authors have completed the ICMJE uniform disclosure form (available at http://dx.doi. org/10.21037/atm-20-4297). The authors have no conflicts of interest to declare.

Ethical Statement: The authors are accountable for all aspects of the work in ensuring that questions related to the accuracy or integrity of any part of the work are appropriately investigated and resolved. The study was conducted in accordance with the Declaration of Helsinki (as revised in 2013), and was reviewed and approved by the ethics committee of Changhai Hospital. All patients had signed informed consent.
Open Access Statement: This is an Open Access article distributed in accordance with the Creative Commons Attribution-NonCommercial-NoDerivs 4.0 International License (CC BY-NC-ND 4.0), which permits the noncommercial replication and distribution of the article with the strict proviso that no changes or edits are made and the original work is properly cited (including links to both the formal publication through the relevant DOI and the license). See: https://creativecommons.org/licenses/by-nc-nd/4.0/.

\section{References}

1. Fitzmaurice C, Abate D, Abbasi N, et al. Global, Regional, and National Cancer Incidence, Mortality, Years of Life Lost, Years Lived With Disability, and DisabilityAdjusted Life-Years for 29 Cancer Groups, 1990 to 2017: A Systematic Analysis for the Global Burden of Disease Study. JAMA Oncol 2019;5:1749-68.

2. Travis WD. The $2015 \mathrm{WHO}$ classification of lung tumors. Der Pathologe 2014;35:188.

3. Arenberg D. Update on screening for lung cancer. Transl Lung Cancer Res 2019;8:S77-87.

4. Gettinger S, Horn L, Jackman D, et al. Five-Year FollowUp of Nivolumab in Previously Treated Advanced NonSmall-Cell Lung Cancer: Results From the CA209-003 Study. J Clin Oncol 2018;36:1675-84.

5. Mansfield AS, Aubry MC, Moser JC, et al. Temporal and spatial discordance of programmed cell death-ligand 1 expression and lymphocyte tumor infiltration between paired primary lesions and brain metastases in lung cancer. Ann Oncol 2016;27:1953-8.

6. Zhou J, Gong Z, Jia Q, et al. Programmed death ligand 1 expression and $\mathrm{CD} 8+$ tumor-infiltrating lymphocyte density differences between paired primary and brain metastatic lesions in non-small cell lung cancer. Biochemical and Biophysical Research Communications 2018;498:751-7.

7. Xia L, Huang $\mathrm{H}$, Xiao $\mathrm{H}$, et al. Utilization of combined PD-L1 expression and neutrophil-to-lymphocyte ratio prior to surgery as a prognostic factor in non-small cell lung cancer with brain metastasis. Transl Cancer Res 2019;8:2864-77.

8. Schumacher TN, Schreiber RD. Neoantigens in cancer immunotherapy. Science 2015;348:69-74.

9. Maleki Vareki S. High and low mutational burden tumors versus immunologically hot and cold tumors and response to immune checkpoint inhibitors. J Immunother Cancer 2018;6:157. 
10. Gandara DR, Paul SM, Kowanetz M, et al. Bloodbased tumor mutational burden as a predictor of clinical benefit in non-small-cell lung cancer patients treated with atezolizumab. Nat Med 2018;24:1441-8.

11. Masciale V, Grisendi G, Banchelli F, et al. Correlating tumor-infiltrating lymphocytes and lung cancer stem cells: a cross-sectional study. Ann Transl Med 2019;7:619.

12. Mandal R, Samstein RM, Lee KW, et al. Genetic diversity of tumors with mismatch repair deficiency influences antiPD-1 immunotherapy response. Science 2019;364:485-91.

13. Blank CU, Haanen JB, Ribas A, et al. CANCER IMMUNOLOGY. The "cancer immunogram". Science 2016;352:658-60.

14. Botticelli A, Salati M, Di Pietro FR, et al. A nomogram to predict survival in non-small cell lung cancer patients treated with nivolumab. J Transl Med 2019;17:99.

15. Wu Y, Chen Y, Yang X, et al. Neutrophil-to-lymphocyte ratio (NLR) and platelet-to-lymphocyte ratio (PLR) were associated with disease activity in patients with systemic lupus erythematosus. International Immunopharmacology 2016;36:94-9.

16. Lang D, Horner A, Brehm E, et al. Early serum tumor marker dynamics predict progression-free and overall survival in single PD-1/PD-L1 inhibitor treated advanced NSCLC-A retrospective cohort study. Lung Cancer 2019;134:59-65.

17. Naqash AR, Stroud CRG, Butt MU, et al. Co-relation of overall survival with peripheral blood-based inflammatory biomarkers in advanced stage non-small cell lung cancer treated with anti-programmed cell death-1 therapy: results from a single institutional database. Acta Oncol 2018;57:867-72.

18. Alba AC, Agoritsas T, Walsh M, et al. Discrimination and Calibration of Clinical Prediction Models: Users' Guides to the Medical Literature. Jama 2017;318:1377-84.

19. Moons KG, Altman DG, Reitsma JB, et al. New Guideline for the Reporting of Studies Developing, Validating, or Updating a Multivariable Clinical Prediction Model: The TRIPOD Statement. Adv Anat Pathol 2015;22:303-5.

20. Herbst RS, Baas P, Kim DW, et al. Pembrolizumab versus docetaxel for previously treated, PD-L1-positive, advanced non-small-cell lung cancer (KEYNOTE-010): a randomised controlled trial. Lancet 2016;387:1540-50.

21. Rittmeyer A, Barlesi F, Waterkamp D, et al. Atezolizumab versus docetaxel in patients with previously treated non-small-cell lung cancer (OAK): a phase 3, openlabel, multicentre randomised controlled trial. Lancet 2017;389:255-65.

22. Yu Y, Zeng D, Ou Q, et al. Association of Survival and
Immune-Related Biomarkers With Immunotherapy in Patients With Non-Small Cell Lung Cancer: A Metaanalysis and Individual Patient-Level Analysis. JAMA Netw Open 2019;2:e196879.

23. Ruiz-Patiño A, Arrieta O, Cardona AF, et al. Immunotherapy at any line of treatment improves survival in patients with advanced metastatic non-small cell lung cancer (NSCLC) compared with chemotherapy (QuijoteCLICaP). Thorac Cancer 2020;11:353-61.

24. Rizvi NA, Hellmann MD, Snyder A, et al. Cancer immunology. Mutational landscape determines sensitivity to PD-1 blockade in non-small cell lung cancer. Science 2015;348:124-8.

25. Chan LC, Li CW, Xia W, et al. IL-6/JAK1 pathway drives PD-L1 Y112 phosphorylation to promote cancer immune evasion. J Clin Invest 2019;129:3324-38.

26. Vokes EE, Ready N, Felip E, et al. Nivolumab versus docetaxel in previously treated advanced non-small-cell lung cancer (CheckMate 017 and CheckMate 057): 3-year update and outcomes in patients with liver metastases. Ann Oncol 2018;29:959-65.

27. Crispe IN. Hepatic T cells and liver tolerance. Nat Rev Immunol 2003;3:51-62.

28. Bamboat ZM, Stableford JA, Plitas G, et al. Human liver dendritic cells promote $\mathrm{T}$ cell hyporesponsiveness. J Immunol 2009;182:1901-11.

29. Holdenrieder S, Wehnl B, Hettwer K, et al. Carcinoembryonic antigen and cytokeratin-19 fragments for assessment of therapy response in non-small cell lung cancer: a systematic review and meta-analysis. Br J Cancer 2017;116:1037-45.

30. Noonan SA, Patil T, Gao D, et al. Baseline and OnTreatment Characteristics of Serum Tumor Markers in Stage IV Oncogene-Addicted Adenocarcinoma of the Lung. J Thorac Oncol 2018;13:134-8.

31. Sertić Milić H, Franjević A, Bubanović G, et al. Size, edge, and stage of NSCLC determine the release of CYFRA 21-1 in bloodstream. Wien Klin Wochenschr 2015;127:465-71.

(English Language Editor: J. Chapnick)

Cite this article as: Chai R, Fan Y, Zhao J, He F, Li J, Han Y. Prognostic nomogram on clinicopathologic features and serum indicators for advanced non-small cell lung cancer patients treated with anti-PD-1 inhibitors. Ann Transl Med 2020;8(17):1078. doi: 10.21037/atm-20-4297 